\title{
Investigation of the association between vitiligo and fasting blood sugar
}

Farrokh Rad ${ }^{1}$, Ebrahim Ghaderi ${ }^{2}$, Bahram Nikkhoo ${ }^{3}$, Mohammad Aziz Rasouli ${ }^{4}$

1. Department of Dermatology, Faculty of Medicine, Kurdistan University of Medical Sciences, Sanandaj, Iran, ORCID CD: 0000-0002-2532-5819

2. Social Determinants of Health Research Center, Research Institute for Health Development, Kurdistan University of Medical Sciences, Sanandaj, Iran

3. Department of Pathology, Faculty of Medicine, Kurdistan University of Medical Sciences, Sanandaj, Iran Iran, Tel: 08733161392, E-mail: Dr.b.nikkhoo@gmail.com

4. Clinical Research Development, Unit Kowsar Hospital, Kurdistan University of Medical Sciences, Sanandaj,

\section{ABSTRACT}

Background and Aim: Vitiligo is a dermatologic disorder which has autoimmune and genetic basis. Different studies in the world have shown relationship of vitiligo with autoimmune disorders. The purpose of this study was to investigate the association between vitiligo and fasting blood sugar (FBS).

Materials and Methods: This cross sectional study was performed between 2016 and 2019 and included 102 patients with vitiligo. Diagnosis of vitiligo was made by our dermatologist and FBS levels of the participants were measured.

Results: Mean age of the patients and duration of the disease were $39.7 \pm 15.8$ years and $56.1 \pm 34.1$ months respectively. $45(\% 44)$ patients were male, the disorder was most frequent in the housewives. The most common site of involvement was genitalia which was affected in $52(\% 31)$ cases, and the least common site was oral mucosa which was involved in 6 patients (\%3.6). Only 4 (\%2.7) patients had family history of vitiligo.

Conclusions: The results showed that there were no significant association between vitiligo and variables of FBS, gender, occupation, site of involvement, family history of vitiligo, history of using medications and also any other disease.

Keywords: Vitiligo, Fasting blood sugar, Kurdistan

Received: Dec30,2018

Accepted: Aug31,2019

How to cite the article: Farrokh Rad, Ebrahim Ghaderi, Bahram Nikkhoo, Mohammad Aziz Rasouli . Investigation of the association between vitiligo and fasting blood sugar. SJKU 2019; 24 (5): 77-82

Copyright (C) 2018 the Author (s). Published by Kurdistan University of Medical Sciences. This is an open access article distributed under the terms of the Creative Commons Attribution-Non Commercial License 4.0 (CCBY$\mathrm{NC}$ ), where it is permissible to download, share, remix, transform, and buildup the work provided it is properly cited. The work cannot be used commercially without permission from the journal. 


\section{بررسى ارتباط بيمارى ويتيليكو با ميزان قند خون}

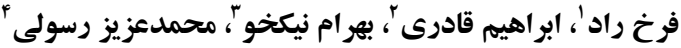

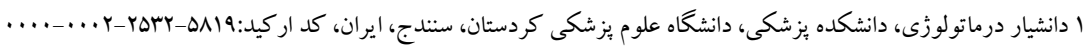

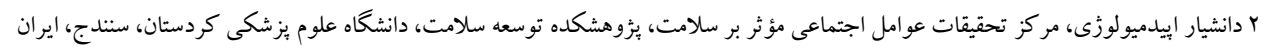

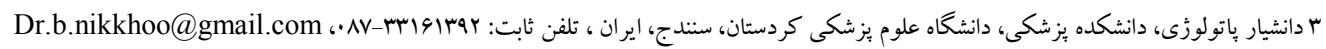

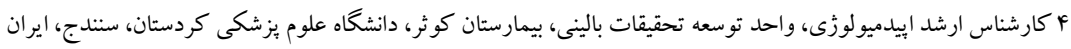

جكيله

زمينه و هدف: ويتيليكو يكك بيمارى بوستى است كه مىتواند به صورت زنتيكك ا-Y درصد جمعيـت عمـومى را بـدون در نظر

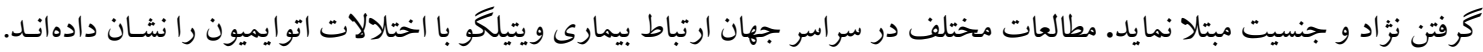

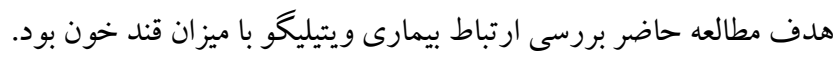

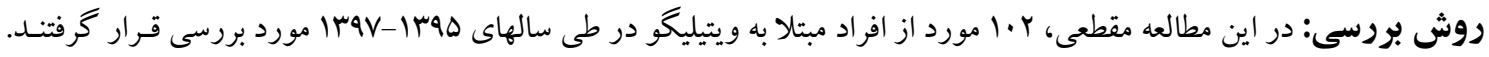

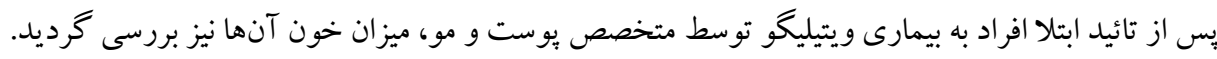

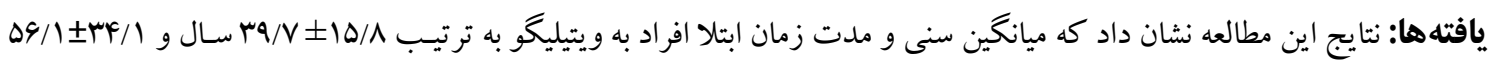

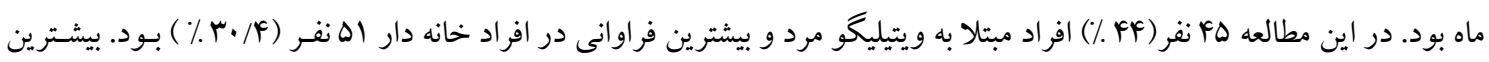

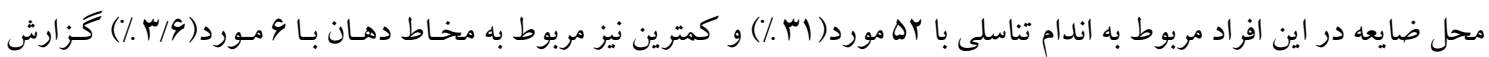

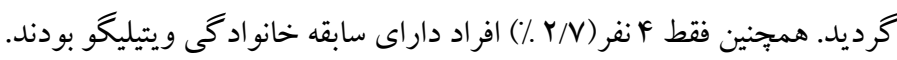

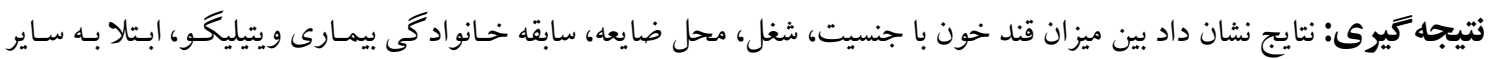
بيمارىها و سابقه مصرف دارو ارتباط آمارى معنى دارى وجود نداشت. كلمات كليدى: ويتيليخو، قند خون، كردستان

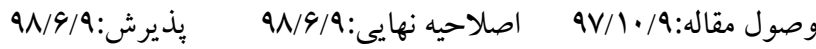


متغيرهـاى دمو گر افيكك شـامل، سـن، جـنس و شـغل آنهـا

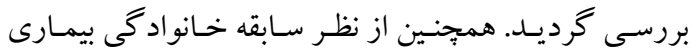

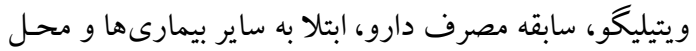

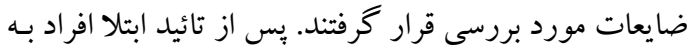

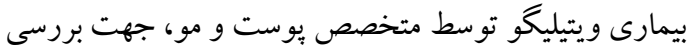

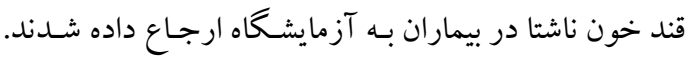

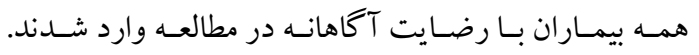
فراوانسى مـوارد ويتيليخـو بـر حسبـ عوامـل مختلـف و نيسز

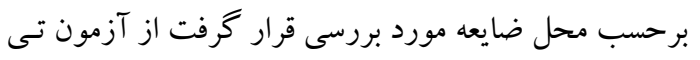

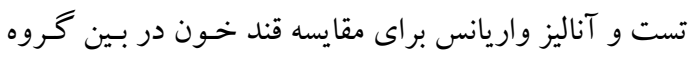

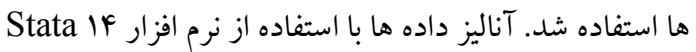
انجام و سطح معنى دارى هـ/ P > در نظر كرفته شد.

يافتهها

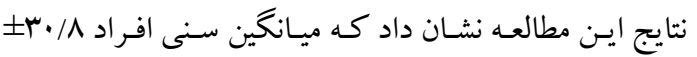

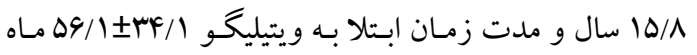

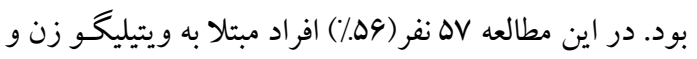

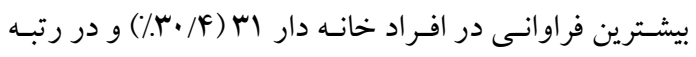

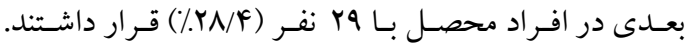

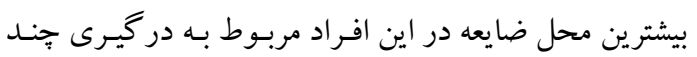

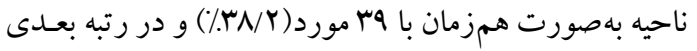

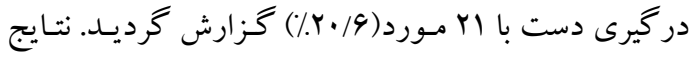

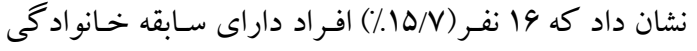

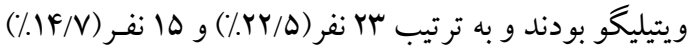

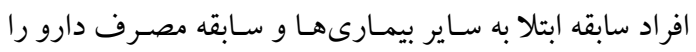

$$
\text { كزارش كردند(جدول (1). }
$$

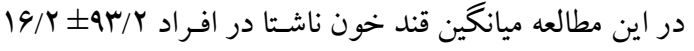

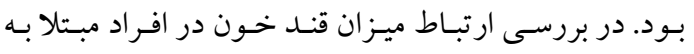

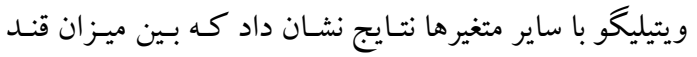

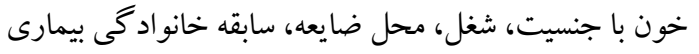

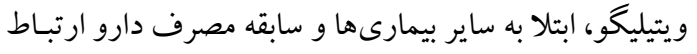

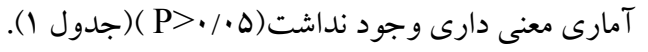

مقدمه ويتيليگو يكك بيمارى بوستى است كـه بـر روى r-1 درصـد

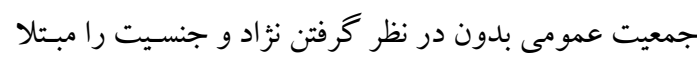

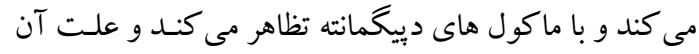
از بين رفتن ملانوسيت هاى فانكشنال است (זو (). تايبهـاى

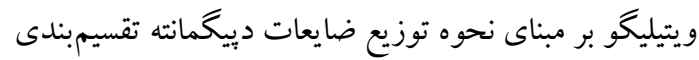

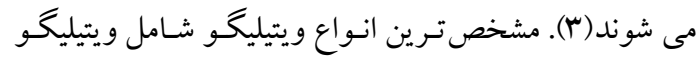

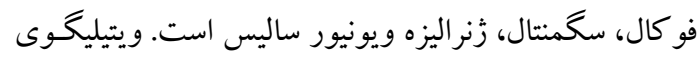

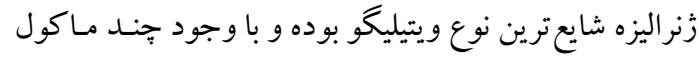
يا تعداد زيادى ماكول هاى بر اكنده در نواحى مختلف بـ بـدن

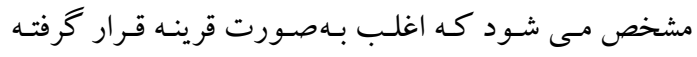

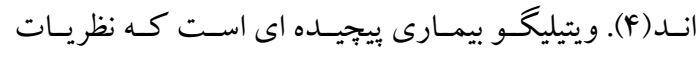

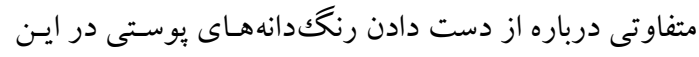
اختلال بيان شده اسـت. مكانيسم هـاى بيشـنهاد شـده شـامل

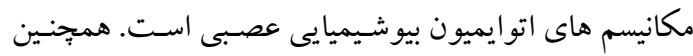

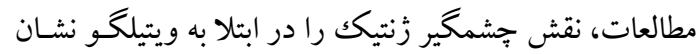
دادند. تشخيص ويتيليكو وابسته به تاريخجه و معاينات بـالينى نئى

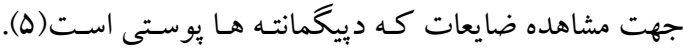
امروزه بيشترين تمر كز محققين بر روى ماهيت اتوايميون اين

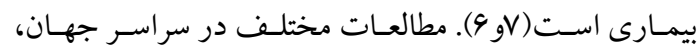

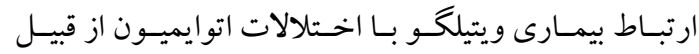

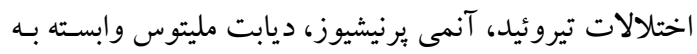

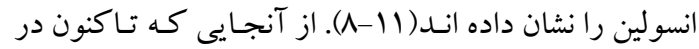

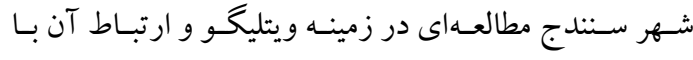

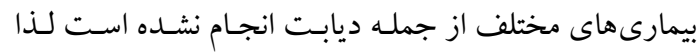

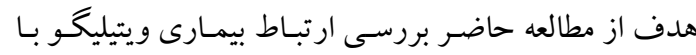

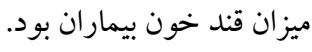

روش بر برسى در اين مطالعه مقطعى(توصيفى - تحليلى)، تعـداد ץ.ا نفـر از

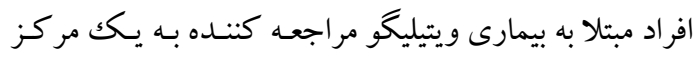

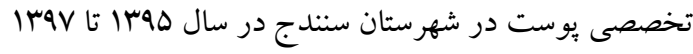

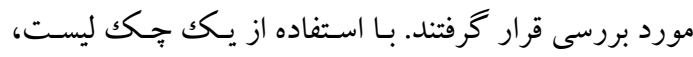


جدول ا. توزيع فراوانى و ميانگين قند خون برحسب جنس، سابقه فاميلى بيمارى، شغل، محل درگيرى و سابقه ابـلال بـه بيمـارى در

\begin{tabular}{|c|c|c|c|}
\hline P-value & 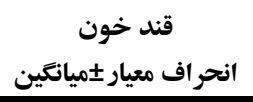 & 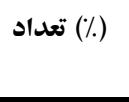 & متغير \\
\hline \multirow[t]{2}{*}{$\cdot / \Delta \wedge \Delta$} & $9 Y / I \pm \mid Y / I$ & $(F F) F \Delta$ & جنسيت \\
\hline & $q r / Y \pm r \cdot / V$ & $(\Delta \varphi) \Delta V$ & زن - ت ان \\
\hline \multirow[t]{2}{*}{$\cdot / \wedge \Delta \Delta$} & $9 r / 4 \pm 1 \cdot / 9$ & $(10 / V) 19$ & سابقه فاميلى بيمارى \\
\hline & $9 \mu / \uparrow \pm \mid N / r$ & $(\Lambda F / r) \wedge \varepsilon$ & 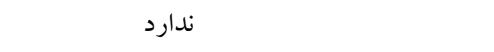 \\
\hline \multirow[t]{5}{*}{.$/ Y 19$} & $q \Delta \pm \mid f / \Delta$ & $(Y / / 9) Y Y$ & كارگر \\
\hline & $99 / \Delta \pm r q$ & $(r \cdot / F) r_{1}$ & خانه دار \\
\hline & $\mathcal{M N} / \mathrm{V} \pm \mathbf{q} / \Delta$ & $(Y N / F) Y q$ & دانش آموز - دانشجو \\
\hline & $\Lambda \Lambda / \Delta \pm r / \Delta$ & $(9 / \Lambda))$ & 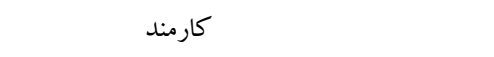 \\
\hline & $\wedge q \pm q / r$ & $(9 / \Lambda))$. & 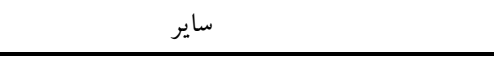 \\
\hline \multirow[t]{7}{*}{$\cdot / \Lambda \cdot r$} & $q \cdot / \uparrow \pm 11 / \Delta$ & $(Y \cdot / 9) Y I$ & محل درگيرى \\
\hline & $q r / r \pm 1 \Lambda / r$ & $(\Delta / 9) 9$ & 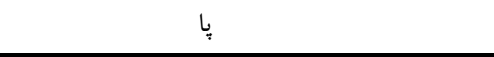 \\
\hline & $q \cdot \pm V / r$ & $(\boldsymbol{G / V}) \mathrm{V}$ & يلك \\
\hline & $\Lambda 9 / \Upsilon \pm 9 / \vee$ & $(r / 9) F$ & 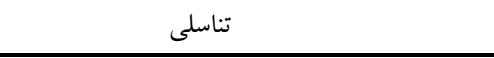 \\
\hline & $|F / \Delta \pm| r / F$ & $(\Delta / 9) 9$ & سينه \\
\hline & $91 \pm V / V$ & $(19 / \mathrm{V}) \mathrm{IV}$ & صورت \\
\hline & 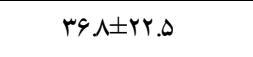 & $(r / / Y) r q$ & در كيرى جند عضو \\
\hline \multirow[t]{2}{*}{. /9Yr } & $9 r / 9 \pm / F / \Lambda$ & $(Y Y / Q) Y r$ & سابقه ابتلا به ساير بيمارى ها دارد \\
\hline & $9 r / 1 \pm \mid N / 1$ & $(V V / \Delta) \Lambda$ & 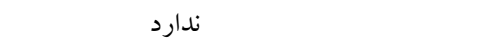 \\
\hline \multirow[t]{2}{*}{$\cdot 19 \cdot 0$} & $Q F / r \pm 1 \Delta / V$ & $(\mid F / V) 10$ & سابقه مصرف دارو \\
\hline & $a r / I \pm I V / \Delta$ & $(\Lambda \Delta / \Gamma) \wedge \vee$ & 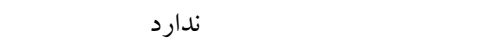 \\
\hline
\end{tabular}

يكديخر متفاوت است. همبجنسين در مطالعه حاضـر اكثريـت افراد مبتلا جنسيت زن داشتند كه با نتايج مطالعات ديخـر نيز

$$
\text { همخوانى داشت (19) }
$$

بر اساس يافته هاى مطالعه حاضـر، بيشـترين محل ضـايعه در

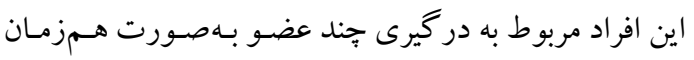

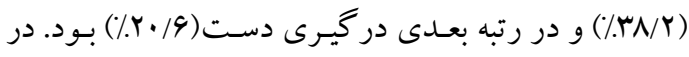
Fatani و همكار ان، شايع ترين محل در زيـرى سـر و كردن(ه) ، در مطالعه Reghu و همكاران اندام هاى تحتانى

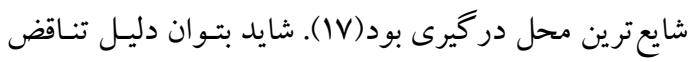

ويتيليكـو يـك اخـتلال زنتيكى كـه بـه علـت از بـين رفـتن ملانوسيت ها در إيدرم يوست ايجاد مى شود(Y) (I). ميـانكين سن رخداد بيمارى بر اساس نتايج مطالعات مختلـف از يـك لـك تا GV سال با ميانكين سنى سو

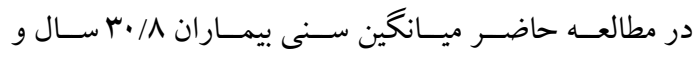

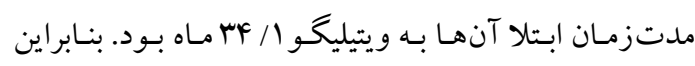
مسى تـوان كفـت ميـانخين سـن بيمـار ان در مطالعـه حاضـر در مقايسه با ساير مطالعات بالاتر است و نشان دهنده ايـن اسـت

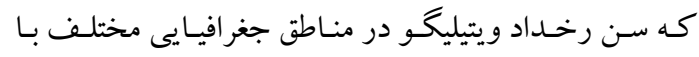




$$
\begin{aligned}
& \text { مليتوس با سن، جنسيت و طول مدت ابتلا به ويتيليكو ارتبـاط } \\
& \text { بين نتايج مطالعات متعدد را اين گونه بيـان كـرد كـه احتمالاً } \\
& \text { معنادارى نداشته اما بـين كسترش ويتيلحَو و ديابـت ارتبـاط } \\
& \text { عو امل زنتيكك و يا محيط مى تواند سبب بروز اين تفـاوت هـا } \\
& \text { در افراد باشد. }
\end{aligned}
$$

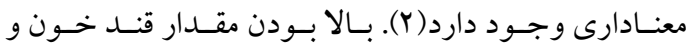

$$
\begin{aligned}
& \text { همجينين بروز بالاى ديابت ممكن اسـت بـه دليـل اخـتلالات }
\end{aligned}
$$

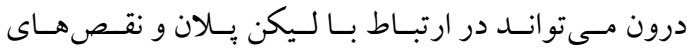

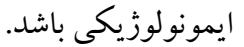

$$
\begin{aligned}
& \text { نتيجه كيرى } \\
& \text { نتايج نشان داد بين ميزان قند خـون و جنسـيت، شـل، محل } \\
& \text { ضـا يعه، سـابقه خـانوادگى بيمـارى ويتيليخـو، ابـتلا بـه سـاير } \\
& \text { بيمارىها و سـابقه مصرف دارو ارتبـاط آمـارى معنى دارى }
\end{aligned}
$$

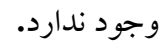

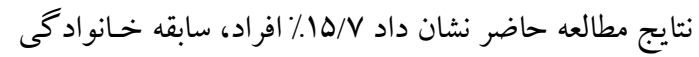

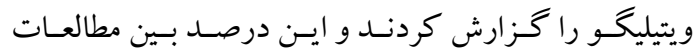

$$
\begin{aligned}
& \text { مختلف از V/D/D د درصد متفاوت است و مى توان كفـت بـا }
\end{aligned}
$$

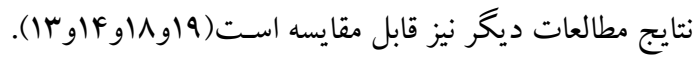

$$
\begin{aligned}
& \text { همانند ساير مطالعـات، يافتـه هـاى مطالعـه حاضـر نيزز، نشـان } \\
& \text { مسدهـــ كـه ويتيليخـو در افراد بـا سـابقه فـاميلى مثبـت رخ }
\end{aligned}
$$

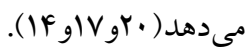

$$
\begin{aligned}
& \text { در اين مطالعه ميانكين قنـد خـون ناشـتا در افـراد ب. به بـود و } \\
& \text { بين ميانگين قند خون با جنسيت، شغل، محـل ضـايعه، سـابقه } \\
& \text { خانوادگى بيمارى ويتيليكو، ابتلا به ساير بيمـارىهـا و سـابقه } \\
& \text { تشكر و قدردانى }
\end{aligned}
$$

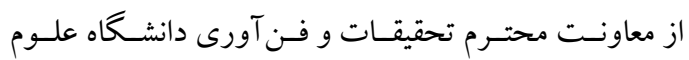

$$
\begin{aligned}
& \text { مصـرف دارو ارتبـاط آمـارى معنسى دارى وجـود نداشـت. }
\end{aligned}
$$

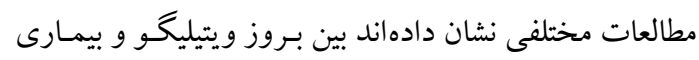

$$
\begin{aligned}
& \text { ديابـت ارتبـاط معنــادارى وجـود دارد( (Y او اY). در مطالعـه } \\
& \text { بزشـكى كردسـتان و دانشـكده بزشـكى بـهـ عنـوان حمايست }
\end{aligned}
$$

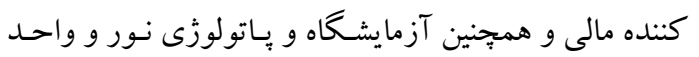

$$
\begin{aligned}
& \text { توسعه تحقيقات بالينى بيمارستان كوثر سـنندج كـه مـا را در } \\
& \text { انجام اين تحقيق يارى نمودنــ تقـــير و تشـكر بـهـ عمـل مى } \\
& \text { آيد } \\
& \text { Gopal } \\
& \text { شاهد ديابتى نبودند اما يافته هاى حاصل از قند خون ناشتا در } \\
& \text { اين بيماران ديابت ميليتوس رادر } 19 \text { درصد از افراد در گروه }
\end{aligned}
$$

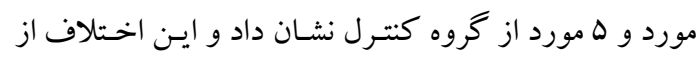

$$
\begin{aligned}
& \text { لحاظ آمارى نيز معنـادار بـود و بيـان كردنـد حضـور ديابـت }
\end{aligned}
$$

\section{References}

1. Ortonne J-P, Passeron T. Vitiligo and other disorders of hypopigmentation. Dermatology. 2008;1(3).

2. Gopal K, Rao GR, Kumar YH. Increased prevalence of thyroid dysfunction and diabetes mellitus in Indian vitiligo patients: A case-control study. Indian dermatology online journal. 2014;5(4):456.

3. Anbar T, Westerhof W, Abdel-Rahman A, El-Khayyat M. Evaluation of the effects of NB-UVB in both segmental and non-segmental vitiligo affecting different body sites. Photodermatology, photoimmunology \& photomedicine. 2006;22(3):157-63.

4. Tayyebi Meibodi N JZ, Mahmodi M, Maleki M and et al. Evaluation of Subtypes of Peripheral Blood Lymphocytes in Patients with Vitiligo. Medical Journal of Mashhad University of Medical Sciences. 2010;52(4):198-202.

5. Fatani M, AlSharif S, Alfif K, Khan A, Hussain W, Banjar A. The clinical patterns of vitiligo "hospital-based study" in Makkah region, Saudi Arabia. Journal of Dermatology \& Dermatologic Surgery. 2014;18(1-2):17-21.

6. Ongenae K, Van Geel N, Naeyaert JM. Evidence for an autoimmune pathogenesis of vitiligo. Pigment Cell Research. 2003;16(2):90-100. 
7. Sehgal VN, Srivastava G. Vitiligo: compendium of clinico-epidemiological features. Indian Journal of Dermatology, Venereology, and Leprology. 2007;73(3):149.

8. McLeod DS, Cooper DS. The incidence and prevalence of thyroid autoimmunity. Endocrine. 2012;42(2):252-65.

9. Fallahi P, Ferrari SM, Ruffilli I, Elia G, Biricotti M, Vita R, et al. The association of other autoimmune diseases in patients with autoimmune thyroiditis: Review of the literature and report of a large series of patients. Autoimmunity reviews. 2016;15(12):1125-8.

10. Huggins RH, Janusz CA, Schwartz RA. Vitiligo: a sign of systemic disease. Indian Journal of Dermatology, Venereology, and Leprology. 2006;72(1):68.

11. Schallreuter K, Lemke R, Brandt O, Schwartz R, Westhofen M, Montz R, et al. Vitiligo and other diseases: coexistence or true association? Dermatology. 1994;188(4):269-75.

12. Gandhi S, Shamanur M, Shashikiran A, Kusagur M, Bhaskar V. A study of clinicoepidemiological and dermoscopic patterns of vitiligo in pediatric age group. Indian Journal of Paediatric Dermatology. 2017;18(4):292.

13. Shajil E, Agrawal D, Vagadia K, Marfatia Y, Begum R. Vitiligo: clinical profiles in Vadodara, Gujarat. Indian Journal of Dermatology. 2006;51(2):100.

14. Shankar DK, Shashikala K, Madala R. Clinical patterns of vitiligo and its associated co morbidities: A prospective controlled cross-sectional study in South India. Indian dermatology online journal. 2012;3(2):114.

15. Arýcan O, Koç K, Ersoy L. Clinical characteristics in 113 Turkish vitiligo patients. Acta Dermatovenerol Alp Pannonica Adriat. 2008;17(3):129-32.

16. Karadag AS, Tutal E, Ertugrul DT. Insulin resistance is increased in patients with vitiligo. Acta dermato-venereologica. 2011;91(5):541-4.

17. Reghu R, James E. Epidemiological profile and treatment pattern of vitiligo in a tertiary care teaching hospital. Int J Pharm Sci. 2011;3:137-41.

18. Niang S, Ndiaye M, Ly F, Diallo M, Bouksani S, Diop A, et al. The vitiligo in Senegal. ISRN dermatology. 2012;2012.

19. Singh S, Pandey SS. Epidemiological profile of vitiligo in Northern India. Journal of Applied Pharmaceutical Science. 2011;1(10):211.

20. Gopal K, Rao GRR, Kumar YHK, Rao MA, Vasudev P. Vitiligo: a part of a systemic autoimmune process. Indian Journal of Dermatology, Venereology, and Leprology. 2007;73(3):162.

21. Perez MI, Kohn SR. Cutaneous manifestations of diabetes mellitus. Journal of the American Academy of Dermatology. 1994;30(4):519-31.

22. Mubki T, Alissa A, Mulekar S, Albargawi S, Youssef M, AlJasser M. Association of vitiligo with anemia, vitamin B12 deficiency, diabetes mellitus, and thyroid dysfunction in Saudi Arab patients: A case control study. Journal of Dermatology \& Dermatologic Surgery. 2017;21(2):72-6.

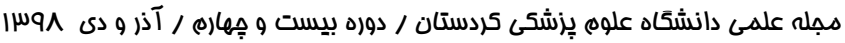

